\title{
Usefulness of FDG, MET and FLT-PET Studies for the Management of Human Gliomas
}

\author{
Keisuke Miyake, Aya Shinomiya, Masaki Okada, Tetsuhiro Hatakeyama, \\ Nobuyuki Kawai, and Takashi Tamiya
} Department of Neurological Surgery, Faculty of Medicine, Kagawa University, 1750-1 Ikenobe, Miki-cho, Kita-gun,
Kagawa 761-0793, Japan

Correspondence should be addressed to Keisuke Miyake, keisuke@kms.ac.jp

Received 5 January 2012; Accepted 7 February 2012

Academic Editor: David J. Yang

Copyright ( $\odot 2012$ Keisuke Miyake et al. This is an open access article distributed under the Creative Commons Attribution License, which permits unrestricted use, distribution, and reproduction in any medium, provided the original work is properly cited.

\begin{abstract}
The use of positron imaging agents such as FDG, MET, and FLT is expected to lead the way for novel applications toward efficient malignancy grading and treatment of gliomas. In this study, the usefulness of FDG, MET and FLT-PET images was retrospectively reviewed by comparing their histopathological findings. FDG, MET, and FLT-PET were performed in 27 patients with WHO grade IV, 15 patients with WHO grade III, and 12 patients with WHO grade II during 5.5 years. The resulting PET images were compared by measuring SUVs and T/N ratios (tumor to normal tissue ratios). Although there were no significant differences in FDG-PET, there were significant differences in the T/N ratios in the MET-PET between WHO grades II and IV and in the FLT-PET between the WHO grades III and IV. In glioblastoma patients, the SUVs of the areas depicted by MRI in the MET-PET were different from those SUVs in the FLT-PET. Importantly, the areas with high SUVs in both MET-PET and FLT-PET were also high in Ki-67 index and were histologically highly malignant. PET imaging is a noninvasive modality that is useful in determining a tumor area for removal as well as improving preoperative diagnosis for gliomas.
\end{abstract}

\section{Introduction}

Morphological imaging using magnetic resonance imaging (MRI) is the most commonly used method for obtaining tumor information. Gd-enhanced T1-weighted MRI provides anatomical imaging with hyperintense neovascularization enclosed in a hypointense region of central necrosis. In addition, fluid-attenuated IR (FLAIR) detects the surrounding edema associated with infiltrating tumor cells [1]. The use of positron emission tomography (PET), an imaging modality providing metabolic and molecular information, can improve diagnostic procedures in malignant brain tumors. 2-Deoxy-2- $\left[{ }^{18} \mathrm{~F}\right]$ fluoro-D-glucose (FDG), a commonly used tracer for neoplasm detection, exhibits limited utility in brain tumor imaging, because the uptake is nonspecific and can occur in any region with increased metabolic activity. Moreover, nonspecific FDG uptake can be increased by some inflammatory diseases. In addition, FDGPET is a marker of glycolytic metabolism and not cellular proliferation. The information it provides is therefore complementary to other imaging techniques [2-5].

L-Methyl- ${ }^{11} \mathrm{C}$-methionine (MET) is a well-established PET radiotracer for brain tumor detection and tumor delineation $[6,7]$. Amino acids, including MET, readily cross the intact blood-brain barrier (BBB) through neutral amino acid transporters and are incorporated into the area with active tumor [8]. It has been shown that MET uptake in gliomas significantly correlates with the WHO tumor grade and cell proliferation determined by Ki-67 index [4, 9-11]. However, increased MET uptake in nonneoplastic lesions including inflammation, infarction, and hemorrhage may result in false positives $[12,13]$. Also, the short half-life of ${ }^{11} \mathrm{C}(20 \mathrm{~min})$ and rapid in vivo degradation make MET-PET less useful for routine clinical use.

A fluorinated thymidine analog, $3^{\prime}$-deoxy- $3^{\prime}-\left[{ }^{18} \mathrm{~F}\right]$ fluorothymidine (FLT), has emerged as a promising PET tracer for evaluating tumor-proliferating activity in various malignant brain tumors $[3,14,15]$. FLT is phosphorylated 
by thymidine kinase-1 (TK1), a principle enzyme in the salvage pathway of DNA synthesis, and trapped inside the cells. Phosphorylated FLT appears resistant to degradation and is suitable for imaging with PET. The application of FLT phosphorylation as a marker of cell proliferation is based on the assumption that cellular FLT trapping is a representation of thymidine incorporation into DNA [16, 17]. Since FLT uptake in the normal brain tissue is very low, FLT-PET provides a low-background brain image, making it an ideal PET tracer for the imaging of brain tumors. FLT-PET has been found useful for noninvasive grading of gliomas [18]. In addition, FLT-PET has been utilized in the prognostic assessment and evaluation of treatment response in malignant gliomas [19].

Despite the superior features MET and FLT offer as glioma cell tracers, they are also associated with several problems. The cut-off value of normal versus tumor cell infiltration is difficult to determine. Therefore, the purpose of this prospective study was to clarify the individual and combined roles of FDG-PET, MET-PET, and FLT-PET in tumor detection, noninvasive grading, and assessment of cellular proliferation rate in 54 newly diagnosed histologically verified gliomas of different grades.

\section{Materials and Methods}

2.1. Patients. From April 2006 through October 2011, 54 patients with newly diagnosed gliomas (23 men and 31 women; mean age: $52.0 \pm 18.1 \mathrm{y}$; range: $22-86 \mathrm{y})$ were enrolled in this study (Table 1). The use of FDG, MET, and FLT as a PET tracer was approved by the Kagawa University School of Medicine Human Subjects Ethics Committee, and informed written consent was obtained from all patients who participated in this study. Histopathology was performed on tissue specimens obtained by biopsy or resection. All tumors were graded by the World Health Organization (WHO) grading system (malignancy scale) for CNS tumors. Tumor types and grades were distributed as follows and listed in Table 1: WHO grade II diffuse astrocytoma $(n=$ 9), WHO grade II oligoastrocytoma $(n=2)$, WHO grade II oligodendroglioma $(n=1)$, WHO grade III anaplastic astrocytoma $(n=11)$, anaplastic oligoastrocytoma $(n=3)$, WHO grade III anaplastic oligodendroglioma $(n=1)$, WHO grade IV glioblastoma multiforme $(n=26)$, WHO grade IV gliosarcoma $(n=1)$.

2.2. Proliferation Analysis. Surgical specimens were fixed in $10 \%$ formalin and embedded in paraffin. Hematoxylinand-eosin-stained specimens were checked to determine the histological tumor type. The cellular proliferation activity of the tumor was determined by measuring the Ki-67 proliferation index obtained by immunohistochemical staining with anti-Ki-67/MIB-1 antibody (Dako, Tokyo, Japan). Immunohistochemical slides were examined at high-power magnification $(\times 400)$. The percentage of tumor cells which stained positively for Ki-67 antigen was measured in the area containing the largest number of positive tumor cells and was regarded as representative of the tumor proliferation activity.
2.3. PET Examination. PET studies were performed using an ECAT EXACT HR + scanner and a Biograph mCT 64 (Siemens/CTI, Knoxville, TN, USA) in three-dimensional acquisition mode. The image system enabled the simultaneous acquisition of 51 transverses per field of view (FOV), with intersection spacing of $3 \mathrm{~mm}$, for a total axial FOV of $15 \mathrm{~cm}$. The in-plane (transverse) reconstructed resolution was $4.7 \mathrm{~mm}$ full-width at half-maximum (FWHM) in the brain FOV. Images were reconstructed using the filtered backprojection method with a Hanning filter (kernel FWHM $10 \mathrm{~mm}$, cutoff frequency 0.4 cycle/projection element) to generate $128 \times 128$ matrices.

PET radiotracers were produced using the HM-18 cyclotron (Sumitomo Heavy Industries, Tokyo, Japan). FLT was synthesized using the method described by Shields et al. [16], and the radiochemical purity of the produced FLT was $>95 \%$. MET was produced by proton bombardment of ${ }^{14} \mathrm{~N}_{2}$. The resultant ${ }^{11} \mathrm{CO}_{2}$ was reduced to ${ }^{11} \mathrm{C}$-methanol by lithium aluminum hydride and subsequently converted to ${ }^{11} \mathrm{C}-\mathrm{CH}_{3} \mathrm{I}$ by the addition of hydrogen iodide following the modified method described by Ishiwata et al. [20]. The radiochemical purity of the produced MET was $>95 \%$.

Each patient received the FDG, MET, and FLT one by one for a continuous 3-day course before the surgery. For the FDG-PET study, enteral and parental sources of glucose were withheld for at least $6 \mathrm{~h}$ before the examination. No special dietary instructions were given to the patients before the MET and FLT-PET examination. Images were acquired with patients in the supine position, resting, with their eyes closed. Using ${ }^{68} \mathrm{Ge}$ rod sources rotating around the head, transmission images of the brain were obtained for $5 \mathrm{~min}$ for FDG-PET, 3 min for MET-PET, and 5 min for FLT-PET. Transmission scan with ${ }^{68} \mathrm{Ge}$ rod can be replaced to CT attenuation correction in mCT64 system. A dose of 147$295 \mathrm{MBq}$ (mean dose: $208 \pm 39 \mathrm{MBq}$ ) of ${ }^{18} \mathrm{~F}-\mathrm{FDG}, 113-$ $389 \mathrm{MBq}$ (mean dose: $211 \pm 65 \mathrm{MBq}$ ) of ${ }^{11} \mathrm{C}-\mathrm{MET}$, or 129 $236 \mathrm{MBq}$ (mean dose: $161 \pm 25 \mathrm{MBq}$ ) of ${ }^{18} \mathrm{~F}$-FLT was injected intravenously. Regional emission images of the brain were obtained for $5 \mathrm{~min}$, beginning $45 \mathrm{~min}$ after the ${ }^{18} \mathrm{~F}-\mathrm{FDG}$ injection; for $5 \mathrm{~min}$, beginning $10 \mathrm{~min}$ after the ${ }^{11} \mathrm{C}$-MET injection; for $10 \mathrm{~min}$, beginning $40 \mathrm{~min}$ after the ${ }^{18} \mathrm{~F}$-FLT injection.

2.4. Image Analysis. FDG, MET, and FLT uptake in the brain tumor were semiquantitatively assessed by evaluating the standardized uptake value (SUV). A region of interest (ROI) was set manually by an observer around the hottest area of each lesion. The maximum value of SUV (SUV max) was regarded as the representative value of each tumor. To calculate the tumor-to-normal tissue count density $(\mathrm{T} / \mathrm{N})$ ratios, the ROI was set on the normal brain parenchyma (usually contralateral normal cerebral tissue excluding ventricles) and the mean value of SUV (SUV mean) was calculated. The $\mathrm{T} / \mathrm{N}$ ratio was determined by dividing the SUV max of the tumor by the SUV mean of the normal brain tissue. The PET and MRI datasets were transferred to a LINUX workstation. Co-registration of FDG-PET/METPET/FLT-PET/MRI was undertaken on the workstation with a commercial software package (Dr. View/LINUX, version 
TAble 1: Patient characteristics and semiquantitative PET results for FDG, MET, and FLT. Dig: histological diagnosis, DA: diffuse astrocytoma, OA: oligoastrocytoma, O: oligodendroglioma, AA: anaplastic astrocytoma, AOA: anaplastic oligoastrocytoma, AO: anaplastic oligodendroglioma, GBM: glioblastoma multiforme, GS: gliosarcoma, SUV max maximum standardized uptake value, T/N ratio tumor-tonormal tissue count density.

\begin{tabular}{|c|c|c|c|c|c|c|c|c|c|c|}
\hline Case & Age & Sex & Dig. & Ki-67 (\%) & $\begin{array}{c}\text { FDG } \\
\text { SUV max }\end{array}$ & FDG T/N & $\begin{array}{c}\text { MET } \\
\text { SUV max }\end{array}$ & MET T/N & $\begin{array}{c}\text { FLT SUV } \\
\max \end{array}$ & FLT T/N \\
\hline 1 & 49 & $\mathrm{f}$ & $\mathrm{DA}$ & 1.75 & 4.12 & 0.403526 & 2.93 & 1.7757576 & 0.13 & 1.1818182 \\
\hline 2 & 42 & $\mathrm{f}$ & $\mathrm{OA}$ & 7.5 & 4.4 & 0.5231867 & 4.37 & 2.5406977 & 0.24 & 1.8461538 \\
\hline 3 & 52 & $\mathrm{f}$ & $\mathrm{DA}$ & 1 & 4.96 & 0.5025329 & 3.34 & 1.9306358 & 0.55 & 3.6666667 \\
\hline 4 & 71 & $\mathrm{~m}$ & DA & 4 & 3.85 & 0.6514382 & 5.28 & 4.3278689 & 0.76 & 5.4285714 \\
\hline 5 & 30 & $\mathrm{~m}$ & DA & 2.5 & 5.65 & 0.495614 & 1.69 & 1 & 0.28 & 1 \\
\hline 6 & 35 & $\mathrm{f}$ & DA & 2.5 & 5.78 & 0.5254545 & 1.57 & 1 & 0.23 & 1 \\
\hline 7 & 30 & $\mathrm{f}$ & DA & 5 & 5.89 & 0.356 & 1.49 & 1.18254 & 0.18 & 1 \\
\hline 8 & 27 & $\mathrm{f}$ & $\mathrm{O}$ & 2.3 & 5.34 & 0.8215385 & 1.38 & 1.5164835 & 0.18 & 1.125 \\
\hline 9 & 49 & $\mathrm{f}$ & $\mathrm{DA}$ & 5 & 2.71 & 0.5441767 & 1.7 & 1.1971831 & 0.22 & 1.047619 \\
\hline 10 & 38 & $\mathrm{f}$ & DA & 5 & 6.07 & 0.497133 & 2.55 & 1.795775 & 0.28 & 1.75 \\
\hline 11 & 37 & $\mathrm{~m}$ & DA & 1 & 23.87 & 2.69 & 4.97 & 2.99 & 0.79 & 2.72 \\
\hline 12 & 36 & $\mathrm{~m}$ & $\mathrm{OA}$ & 7.7 & 8.01 & 1.055336 & 5.26 & 3.094118 & 0.48 & 2.181818 \\
\hline 13 & 34 & $\mathrm{f}$ & $\mathrm{AA}$ & 5 & 6.67 & 0.6778455 & 3.22 & 2.2517483 & 1.15 & 6.7647059 \\
\hline 14 & 58 & $\mathrm{~m}$ & AA & 12.5 & 4.46 & 0.566709 & 3.55 & 2.9583333 & 0.28 & 1.8666667 \\
\hline 15 & 77 & $\mathrm{f}$ & AA & 35 & 6.98 & 1.8082902 & 5.07 & 3.5454545 & 0.9 & 5 \\
\hline 16 & 26 & $\mathrm{~m}$ & $\mathrm{AA}$ & 5 & 4.72 & 0.5777234 & 3.32 & 2.862069 & 0.32 & 2.6666667 \\
\hline 17 & 66 & $\mathrm{f}$ & $\mathrm{AOA}$ & 5 & 3.97 & 0.5321716 & 2.93 & 2.4016393 & 0.58 & 3.0526316 \\
\hline 18 & 60 & $\mathrm{f}$ & $\mathrm{AA}$ & 10 & 7.59 & 1.1587786 & 3.41 & 2.544776 & 0.94 & 3.91667 \\
\hline 19 & 36 & $\mathrm{f}$ & $\mathrm{AA}$ & 6 & 4.27 & 0.5488432 & 4.81 & 2.829412 & 0.37 & 2.176471 \\
\hline 20 & 43 & $\mathrm{f}$ & $\mathrm{AA}$ & 30 & 4.74 & 0.629482 & 8.04 & 4.345946 & 0.84 & 2.470588 \\
\hline 21 & 71 & $\mathrm{~m}$ & $\mathrm{AOA}$ & 50 & 24.02 & 3.1605263 & 5.17 & 4.3445378 & 1.45 & 6.3043478 \\
\hline 22 & 64 & $\mathrm{~m}$ & $\mathrm{AO}$ & 50 & 8.74 & 1.208852 & 8.18 & 6.544 & 2.51 & 9.653846 \\
\hline 23 & 28 & $\mathrm{f}$ & $\mathrm{AOA}$ & 5 & 13.5 & 1.674938 & 9.02 & 5.401198 & 0.98 & 2.227273 \\
\hline 24 & 79 & $\mathrm{f}$ & $\mathrm{AA}$ & 5 & 47.57 & 5.3032329 & 5.96 & 3.8701299 & 0.77 & 5.1333333 \\
\hline 25 & 66 & $\mathrm{f}$ & AA & 12.5 & 6.23 & 0.7634803 & 2.61 & 1.482955 & 0.66 & 2.357143 \\
\hline 26 & 31 & $\mathrm{f}$ & AA & 20 & 23.05 & 2.845679 & 6.49 & 4.570423 & 1.64 & 9.647059 \\
\hline 27 & 45 & $\mathrm{~m}$ & $\mathrm{AA}$ & 7.5 & 14.8 & 1.9865772 & 6.61 & 5.1640625 & 1.06 & 3.2121212 \\
\hline 28 & 51 & $\mathrm{~m}$ & GBM & 25 & 14.86 & 2.006192 & 20.88 & 5.7246377 & 11.18 & 6.0812183 \\
\hline 29 & 22 & $\mathrm{f}$ & GBM & 40 & 12.69 & 1.4859485 & 5.21 & 3.196319 & 3.05 & 11.296296 \\
\hline 30 & 62 & $\mathrm{~m}$ & GBM & 35 & 7.65 & 0.9634761 & 4.55 & 3.64 & 1.19 & 7 \\
\hline 31 & 70 & $\mathrm{f}$ & GBM & 35 & 5.04 & 0.6990291 & 3.44 & 2.3401361 & 1.37 & 6.2272727 \\
\hline 32 & 81 & $\mathrm{f}$ & GBM & 50 & 13.05 & 2.8935698 & 5.69 & 3.6012658 & 3.74 & 15.583333 \\
\hline 33 & 55 & $\mathrm{f}$ & GBM & 35 & 8.99 & 2.5759312 & 5.35 & 3.4294872 & 2.61 & 3.1829268 \\
\hline 34 & 29 & $\mathrm{f}$ & GBM & 50 & 5.5 & 0.607064 & 3.57 & 1.9944134 & 1.66 & 8.7368421 \\
\hline 35 & 77 & $\mathrm{f}$ & GBM & 45 & 4.6 & 1.281337 & 5.51 & 3.3393939 & 2.15 & 8.9583333 \\
\hline 36 & 79 & $\mathrm{~m}$ & GBM & 65 & 9.71 & 2.0442105 & 4.3 & 3.5833333 & 3.68 & 17.52381 \\
\hline 37 & 69 & $\mathrm{f}$ & GBM & 15 & 15.1 & 2.2913505 & 4.98 & 3.254902 & 4.41 & 23.210526 \\
\hline 38 & 86 & $\mathrm{f}$ & GBM & 30 & 10.96 & 1.7734627 & 5.91 & 5.472222 & 2.69 & 15.82353 \\
\hline 39 & 27 & $\mathrm{f}$ & GBM & 30 & 6.51 & 1.4563758 & 3.83 & 2.992188 & 1.53 & 6.652174 \\
\hline 40 & 61 & $\mathrm{~m}$ & GBM & 10 & 6.07 & 1.0049669 & 3.68 & 3.2857143 & 1.96 & 7.5384615 \\
\hline 41 & 53 & $\mathrm{~m}$ & GBM & 25 & 6.61 & 1.0200617 & 3.31 & 3.412371 & 1.7 & 8.5 \\
\hline 42 & 62 & $\mathrm{~m}$ & GBM & 70 & 4.86 & 0.9382239 & 4.54 & 4.09009 & 1.67 & 15.18182 \\
\hline 43 & 67 & $\mathrm{~m}$ & GBM & 30 & 5.8 & 0.9148265 & 2.82 & 2.014286 & 1.45 & 9.0625 \\
\hline 44 & 60 & $\mathrm{~m}$ & GBM & 40 & 5.97 & 0.894615 & 2.9 & 2.788462 & 1.28 & 7.111111 \\
\hline 45 & 54 & $\mathrm{~m}$ & GS & 40 & 6.95 & 0.8742138 & 3.75 & 2.218935 & 2.9 & 12.6087 \\
\hline
\end{tabular}


TABle 1: Continued.

\begin{tabular}{lcccccccccc}
\hline Case & Age & Sex & Dig. & Ki-67 $(\%)$ & $\begin{array}{c}\text { FDG } \\
\text { SUV max }\end{array}$ & FDG T/N & $\begin{array}{c}\text { MET } \\
\text { SUV max }\end{array}$ & MET T/N & $\begin{array}{c}\text { FLT SUV } \\
\text { max }\end{array}$ & FLT T/N \\
\hline 46 & 72 & m & GBM & 50 & 5.55 & 0.9771127 & 3.22 & 2.439394 & 2.28 & 16.28571 \\
47 & 68 & $\mathrm{f}$ & GBM & 60 & 5.94 & 1.1020408 & 4.08 & 2.4 & 2.99 & 17.58824 \\
48 & 53 & $\mathrm{f}$ & GBM & 20 & 4.76 & 0.6979472 & 2.63 & 1.992424 & 1.04 & 6.1176471 \\
49 & 61 & $\mathrm{f}$ & GBM & 70 & 12.2 & 1.75 & 5.64 & 5.271028 & 1.58 & 5.8518519 \\
50 & 37 & $\mathrm{~m}$ & GBM & 20 & 10.31 & 1.19 & 14.08 & 9.7777778 & 1.73 & 6.1785714 \\
51 & 33 & $\mathrm{~m}$ & GBM & 70 & 20.16 & 2.011976 & 8.71 & 4.608466 & 2.85 & 8.6363636 \\
52 & 41 & $\mathrm{f}$ & GBM & 20 & 24.76 & 3.4057771 & 7.04 & 5.1764706 & 2.22 & 17.076923 \\
53 & 26 & $\mathrm{~m}$ & GBM & 80 & 13.32 & 1.5857143 & 5.43 & 3.878571 & 2.59 & 4.177419 \\
54 & 69 & $\mathrm{~m}$ & GBM & 90 & 27.09 & 5.0166667 & 8.86 & 7.5726496 & 5.44 & 34 \\
\hline
\end{tabular}

R2.5, AJS Inc., Tokyo, Japan) with the mutual information method. The software allowed an operator to perform manual intervention; however, required adjustment for coregistration was minimal.

The measured SUV max and T/N ratios of FDG, MET and FLT were compared with the histological diagnoses obtained by biopsy or resection. The relationships between the Ki-67 index and SUV max or T/N ratio of FDG, MET and FLT were evaluated. The relationship between FDG and MET or FLT and MET uptake were evaluated. Regarding the area with a high-intensity signal on the MRI (FLAIR) of each patient, based on a predetermined ROI, the mean SUVs of FDG, MET and FLT were used to compare the values between FDG and MET, between FLT and MET, and between FDG and FLT.

2.5. Statistical Analysis. All parametric data were expressed as mean \pm standard deviation. The Mann-Whitney $U$ test was used to assess the statistical significance in mean volumes across modalities. The Spearman correlation test was used to calculate the correlation coefficient. Statistical analysis was performed using Stat View J-5.0 for Windows.

\section{Results}

3.1. Patient Characteristics and Semiquantitative PET Results for FDG, MET, and FLT. Among the 54 glioma cases, the MET accumulation was observed in 51 cases $(94.4 \%)$ in the MET-PET study and the FLT accumulation was observed in 50 cases $(92.6 \%)$ in the FLT-PET study. Regarding the 3 cases without the MET accumulation and the 4 cases without the FLT accumulation, they were grade II gliomas. All malignant gliomas (grades III and IV) showed accumulation in both studies. Among the cases with T/N ratios higher than 1 in the FDG-PET study, there were 2 cases $(16.7 \%)$ of the grade II gliomas, 8 cases $(53.3 \%)$ of the grade III gliomas, and 17 cases $(63.0 \%)$ of the grade IV gliomas (Table 1). In FDGPET study, it is possible to differentiate gliomas by the WHO grade with the sensitivity of $53.3 \%$, the specificity of $55.6 \%$, the false positive of $44.4 \%$, and the false negative of $46.7 \%$. In MET-PET study, it is possible to differentiate gliomas by WHO grade with the sensitivity of $95.3 \%$, the specificity of $9.1 \%$, the false positive of $90.9 \%$, and the false negative of $4.7 \%$. In MET-PET study, it is possible to differentiate gliomas by WHO grade with the sensitivity of $93.9 \%$, the specificity of $20 \%$, the false positive of $80 \%$, and the false negative of $6.1 \%$.

3.2. Comparison of SUV max and T/N Ratios for FDG, MET, and FLT in Gliomas by WHO Grade. There were no significant differences in the SUV and T/N ratio among each group of the different WHO grades in the FDG-PET study (Figures 1(a) and 1(d)).

SUV of the normal brain in the MET-PET study was $1.52 \pm 0.36(0.92-2.62)$. Regarding the tumors in the METPET study, mean SUV max was $3.04 \pm 1.56$ for the grade II gliomas, $5.22 \pm 2.09$ for the grade III gliomas and $5.12 \pm$ 2.44 for the grade IV gliomas (Figure $1(\mathrm{~b})$ ). $\mathrm{T} / \mathrm{N}$ ratio was $2.03 \pm 1.02$ for the grade II gliomas, $3.67 \pm 1.38$ for the grade III gliomas and $3.76 \pm 1.78$ for the grade IV gliomas (Figure 1(e)). The mean SUV max and $\mathrm{T} / \mathrm{N}$ ratio of the grade IV gliomas were significantly higher than that of the grade II gliomas, while there were no significant differences between the grade II gliomas and grade III gliomas. In the present study, there were some oligodendroglioma cases, which were in grade II, but their SUV max were high and ranged from 4.0 to 6.8 .

SUV of the normal brain in the FLT-PET study was $0.20 \pm 0.05(0.13-0.28)$. Regarding the tumors in the FLTPET study, mean SUV max was $0.36 \pm 0.23$ for the grade II gliomas, $0.96 \pm 0.58$ for the grade III gliomas, and $2.38 \pm 1.07$ for the grade IV gliomas (Figure $1(\mathrm{c})$ ). The mean $\mathrm{T} / \mathrm{N}$ ratio was $1.99 \pm 1.37$ for the grade II gliomas, $4.43 \pm 2.61$ for the grade III gliomas and $11.54 \pm 6.86$ for the grade IV gliomas (Figure 1(f)). There were significant differences in both SUV max and $\mathrm{T} / \mathrm{N}$ ratio between the grade III gliomas and the grade IV gliomas, but, similar to the MET study, there were no significant differences between the grade II gliomas and the grade III gliomas.

3.3. The Correlations between SUV max or $T / N$ Ratio from PET Studies and Ki-67 Index. When the accumulation of MET or FLT within the tumor and the Ki-67 index (an index for proliferation ability of tumor) were compared, linear regression analysis revealed a significant correlation between the Ki-67 index and MET SUV max $(r=0.32, P=0.02)$ 


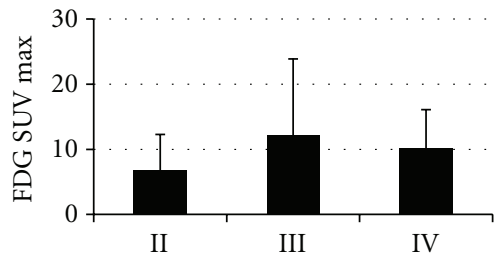

(a)

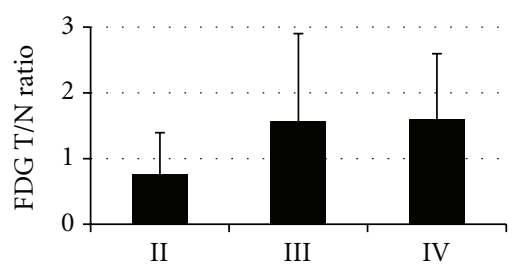

(d)

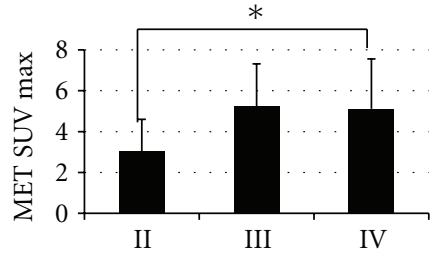

(b)

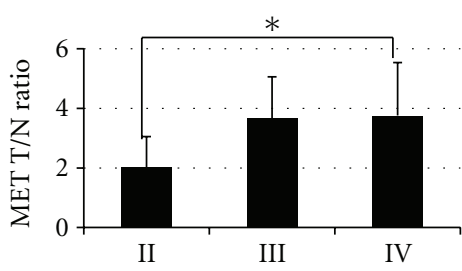

(e)

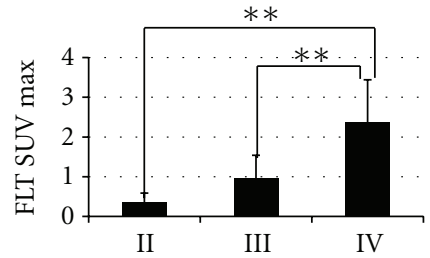

(c)

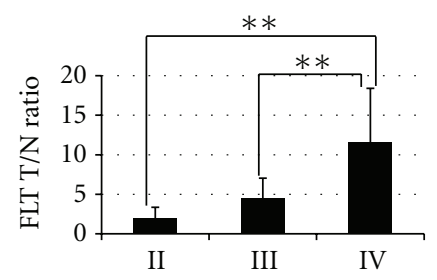

(f)

FIgURe 1: Comparison of SUV max and T/N ratios for FDG (a), (d) MET (b), (e) and FLT (c), (f) in gliomas by WHO grade. There were no significant differences in FDG-PET scan. The differences in MET SUV max and T/N ratio were statistically significant between grades II and IV gliomas $\left({ }^{*} P<0.05\right)$, but not significant between grade II and III gliomas. The differences in FLT SUV max and T/N ratio were statistically significant between grade III and IV gliomas, grade II and grade IV ones $\left({ }^{* *} P<0.01\right)$.

(Figure $2(\mathrm{~b}))$ or MET $\mathrm{T} / \mathrm{N}$ ratio $(r=0.39, P<0.01)$ (Figure 2(e)), as well as FLT SUV $\max (r=0.73, P<0.001)$ (Figure $2(\mathrm{c})$ ) or FLT T/N ratio $(r=0.63, P<0.001)$ (Figure 2(f)). FLT, compared to MET, demonstrates a significantly strong correlation with the proliferation ability. In FDG-PET study, linear regression analysis revealed no correlation between the Ki-67 index and SUV $\max (r=0.16$, $P=0.24)$ (Figure $2(\mathrm{a}))$ or $\mathrm{T} / \mathrm{N}$ ratio $(r=0.33, P=0.02)$ (Figure 2(d)).

3.4. Linear Regression Analysis of T/N Ratio between FDG and MET and between FLT and MET in Gliomas by WHO Grade. Regarding the area with a high intensity signal on the MRI (FLAIR) of each patient, based on a predetermined $\mathrm{ROI}, \mathrm{T} / \mathrm{N}$ ratio of FDG, MET and FLT were used to compare the values between FDG and MET, between FLT and MET and between FDG and FLT. Between FDG and MET, the T/N ratio of the MET tended to be high without correlating with increased $\mathrm{T} / \mathrm{N}$ ratio of the FDG because the oligodendroglioma components were included in the grade II or grade III gliomas (Figures $3(\mathrm{a})$ and $3(\mathrm{~b})$ ). T/N ratio of the FDG tended to be high with correlating with low $\mathrm{T} / \mathrm{N}$ ratio of the MET showing a tendency toward the focus of the epileptic changes (Figures 3(a) and 3(b) black circle). A significant but weak correlation is observed between the individual T/N ratio of FDG and MET in the grade IV gliomas $(r=0.57, P=0.03$ ) (Figure 3(c)). Between FLT and $\mathrm{MET}$, the $\mathrm{T} / \mathrm{N}$ ratio of the MET tended to be high without correlating with increased $\mathrm{T} / \mathrm{N}$ ratio of the FLT because the oligodendroglioma components were included in the grade II or grade III gliomas (Figures $3(\mathrm{~d})$ and $3(\mathrm{e})$ ). We observed the same findings on the correlation between FDG and MET. On the other hand, no significant correlation is observed between the individual T/N ratio of FLT and MET in the grade IV gliomas $(r=0.13, P=0.61)$ (Figure $3(\mathrm{f}))$. However, this relationship between FLT and MET is divided into three parts. Linear regression analysis showed a significant correlation between FLT and MET ( $r=0.21, P=0.008)$ in most areas and these areas appeared as astrocytic tumors (Figure 3(f), red circle). The $\mathrm{T} / \mathrm{N}$ ratio of the MET tended to be high without correlating with increased $\mathrm{T} / \mathrm{N}$ ratio of the FLT $(r=0.53, P=0.014)$, because the oligodendroglioma components were included in the grade IV gliomas (Figure 3(f), blue circle). The $\mathrm{T} / \mathrm{N}$ ratio of the MET tended to be low without correlating with increased $\mathrm{T} / \mathrm{N}$ ratio of the FLT $(r=0.12, P=0.11)$ because the necrotic components were included in the grade IV gliomas (Figure 3(f), green circle). A significant correlation is observed between the individual T/N ratio of FDG and FLT in the grade IV gliomas $(r=0.54, P=0.05)$. Between FDG and FLT, T/N ratio of the FDG tended to be high with correlating with low $\mathrm{T} / \mathrm{N}$ ratio of the FLT showing a tendency toward the focus of the epileptic changes (Figures $3(\mathrm{~g})$ and $3(\mathrm{~h})$ black circle). The $\mathrm{T} / \mathrm{N}$ ratio of the FDG tended to be low without correlating with increased $\mathrm{T} / \mathrm{N}$ ratio of the FLT, because the necrotic components were included in the grade IV gliomas (Figure 3(i), green circle).

3.5. Representative Cases. Figures 4 and 5, respectively, show representative cases of FDG, MET, and FLT-PET studies in anaplastic astrocytoma and glioblastoma.

3.5.1. Case 26: 31-Year-Old Female. She had an onset of convulsion. FLAIR showed a high-intensity lesion (Figure 4(a)), and Gd-enhanced MRI showed slightly enhanced lesion in the right frontal lobe (Figure 4(b)). FDG-PET demonstrated increased FDG uptake within the tumor (Figure 4(c)). MET-PET demonstrated increased MET uptake within the tumor (Figure 4(d)). FLT-PET demonstrated increased FLT uptake within the tumor (Figure 4(e)). Areas of different enhancement and uptake between FDG, MET and FLT are projected on the tumor, in order to perform histological sampling for further correlation during the resection (Figure 4 


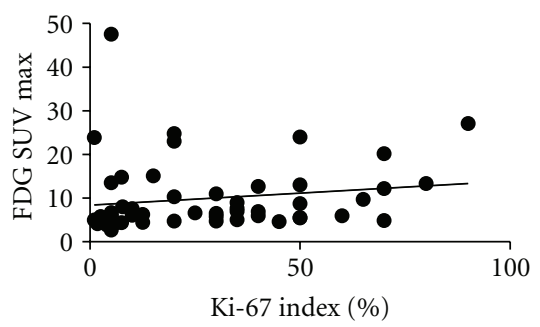

(a)

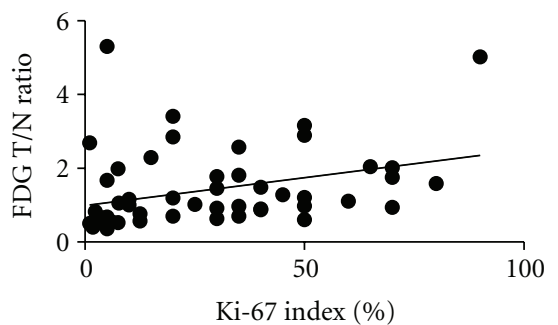

(d)

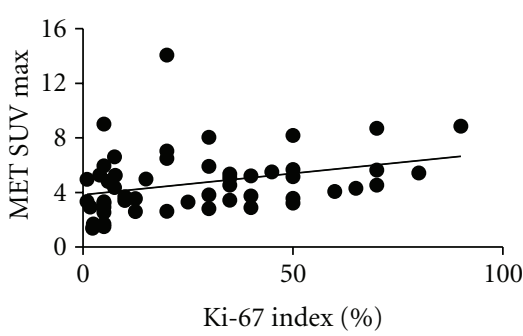

(b)

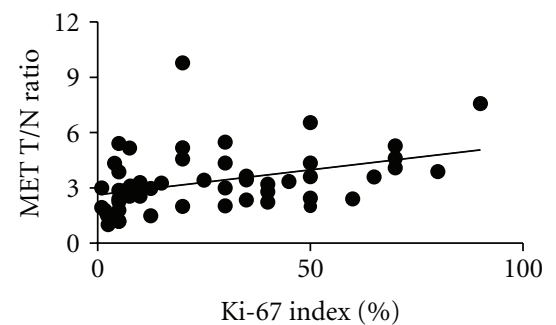

(e)

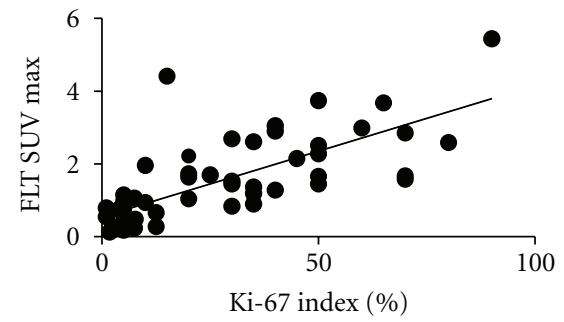

(c)

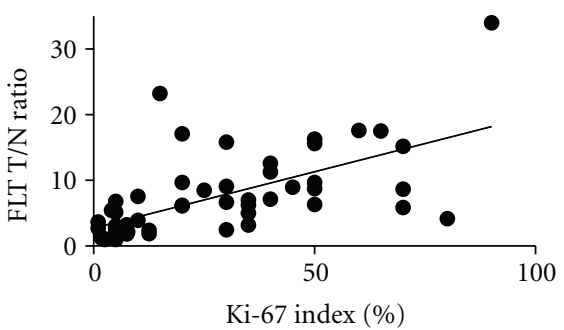

(f)

FIgURE 2: The correlations between SUV max (FDG (a), MET (b), and FLT (c)) and Ki-67 index were determined. Analysis indicates a more significant correlation between the Ki-67 index and FLT SUV $\max (r=0.72, P<0.001)$ than between the index and FDG SUV max $(r=0.16, P=0.24)$ or MET SUV $\max (r=0.32, P=0.02)$. The correlations between T/N ratio (FDG (d), MET (e) and FLT (f) ) and Ki-67 index were determined. Analysis indicates a more significant correlation between the Ki-67 index and FLT T/N ratio $(r=0.63, P<0.001)$ than between the index and FDG T/N ratio $(r=0.33, P=0.02)$ or MET T/N ratio $(r=0.39, P<0.01)$.

yellow arrow, red arrow). Yellow arrow demonstrates anaplastic astrocytoma (Figure $4(\mathrm{f})$ ) within $\mathrm{T} / \mathrm{N}$ ratio of FDG-PET (2.85), MET-PET (4.57) and FLT-PET (9.65). Ki67 index from the specimen indicated by yellow arrow was 20\% (Figure 4(g)). Red arrow demonstrates diffuse astrocytoma area (Figure $4(\mathrm{~h})$ ) within $\mathrm{T} / \mathrm{N}$ ratio of FDG-PET (0.76), MET-PET (2.08) and FLT-PET (4.65). Ki-67 index from the specimen indicated by red arrow was $8 \%$ (Figure 4(i)). She has been treated for anaplastic astrocytoma.

3.5.2. Case 28: 51-Year-Old Male. He experienced an onset of aphasia and Gerstmann's syndrome and developed glioblastoma which was recognized with a ring-like contrast in the left temporo-occipital lobe on the Gd-enhanced MRI (Figure 5(a)). The MRI study did not show a uniformed contrast, and some areas had high-intensity signal while others did not. Similar to the MRI study results, the accumulation was not uniform in MET-PET and FLT-PET studies. This relationship between MET and FLT is divided into three parts, similar to the observation described in Figure 3(f). In the blue arrow area, the contrast by MRI was not strong, but the $\mathrm{T} / \mathrm{N}$ ratio of MET was 4.31 and the $\mathrm{T} / \mathrm{N}$ ratio of FLT was 3.24 with a significant accumulation of MET. In the green arrow area, the contrast by MRI was strong, the $\mathrm{T} / \mathrm{N}$ ratio of $\mathrm{MET}$ was 1.60 , and the T/N ratio of FLT was 5.72 with a significant accumulation of FLT. In the red arrow area, the contrast by MRI was recognized, but the $\mathrm{T} / \mathrm{N}$ ratios of MET and FLT were both high (3.27 and 5.28; resp., Figures 5(b) and 5(c)). He underwent craniotomy for histopathological diagnosis. The diagnosis revealed the blue arrow area mostly with a histological picture of oligodendroglioma (Figure 5(d)) with a Ki-67 index of $30 \%$ (Figure $5(\mathrm{e})$ ) and the green arrow area mainly with a histological picture of necrotic tissues (Figure 5(f)) with a Ki-67 index of less than 10\% (Figure $5(\mathrm{~g})$ ). As for the red arrow area displaying high accumulation of both MET and FLT, this area showed a strong nuclear atypia with megakaryocytes with a high Ki67 index of $70 \%$ (Figures $5(\mathrm{~h})$ and $5(\mathrm{i})$ ). Taken together, increased accumulation of both tracers (MET and FLT) appears to indicate high-grade malignancy.

\section{Discussion}

Accurate tissue diagnosis of glioma is important in order to determine appropriate therapeutic strategy and predict prognosis. Final diagnosis of the grade of malignancy is carried out by surgical histopathological diagnosis. However, due to the diverse nature of gliomas within tumors, some glioma cases tend to be diagnosed less stringent than actual grade of malignancy through pathological diagnosis of a specimen taken from the tumor. The image analysis using PET has an advantage of spatial evaluation of diverse malignant gliomas $[8,21,22]$. FDG has been widely used as a PET tracer for diagnosis of malignant brain tumors. The accumulation of FDG increases in highly malignant tumors, and this method is highly specific as an image analysis and useful for predicting prognosis [23]. However, FDG uptake is high in the normal brain cortex where glucose metabolism is active, and the difference between the normal accumulation and brain tumors may not be detected. In addition, FDG uptake is low in low-grade tumors, and FDG has limitations for detecting the localization of small tumors or accurately identifying tumor area $[5,24,25]$. Extra attention is especially required for diagnosis of gliomas. 


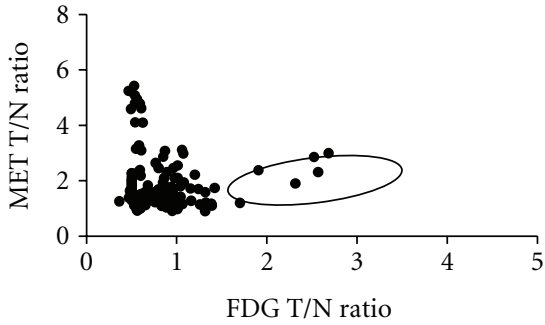

(a)

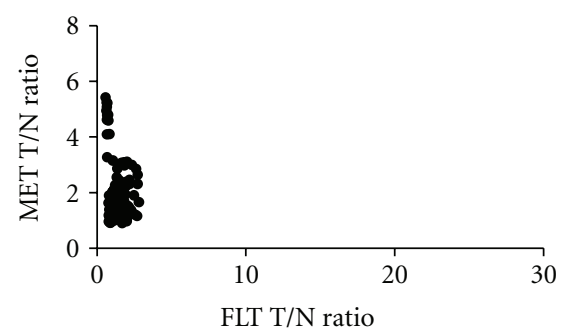

(d)

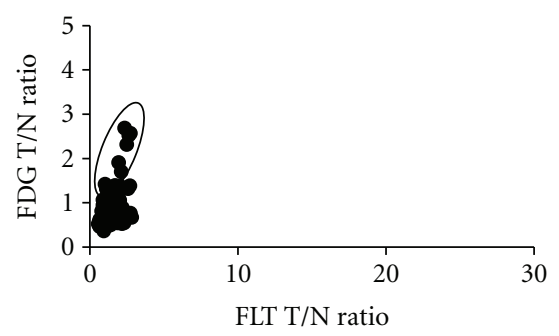

(g)

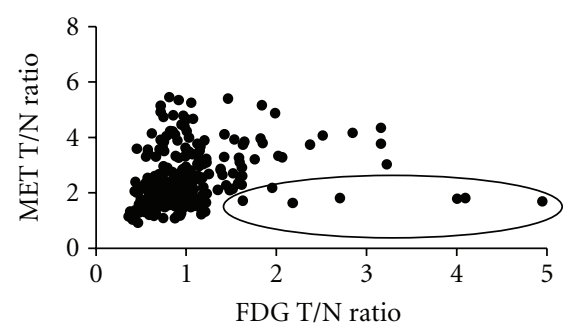

(b)

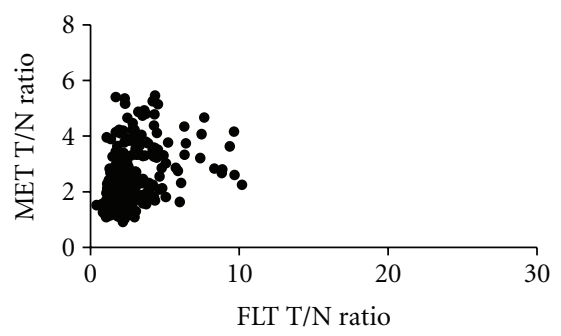

(e)

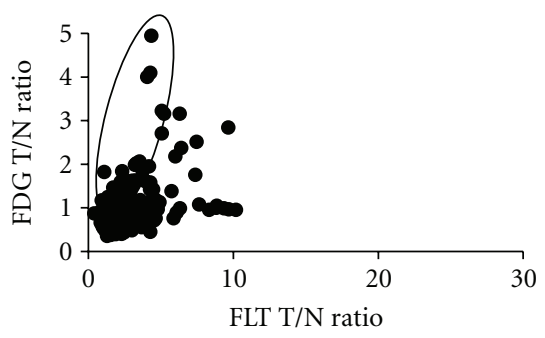

(h)

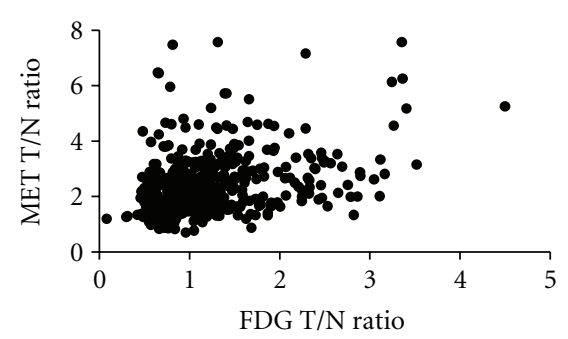

(c)

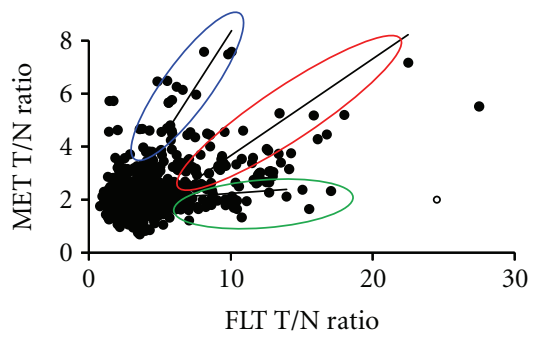

(f)

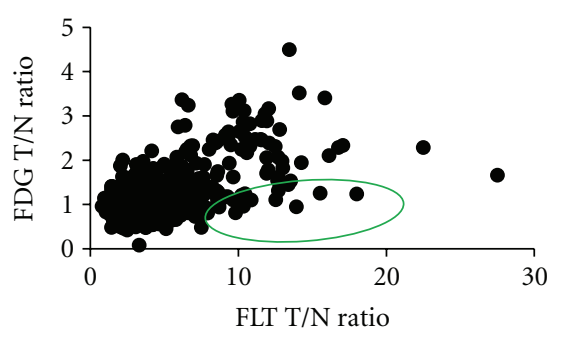

(i)

FIgure 3: Linear regression analysis of T/N ratio between FDG and MET (grade II (a), grade III (b), grade IV (c)), between FLT and MET (grade II (d), grade III (e), grade IV (f)) or between FDG and FLT (grade II (g), grade III (h), grade IV (i)). A significant but weak correlation is observed between the individual T/N ratio of FDG and MET $(r=0.57, P=0.03)$ (c). FDG T/N ratio tended to be high correlating with low MET T/N ratio showing a tendency toward the focus of the epileptic changes (black circle). On the other hand, no significant correlation is observed between the individual T/N ratio of FLT and MET $(r=0.13, P=0.61)$ (f). This relationship between FLT and MET is divided into three parts. Linear regression analysis showed a significant correlation between FLT and MET $(r=0.21, P=0.008)$ in most areas and these areas appeared as astrocytic tumors (red circle). The T/N ratio of the MET tended to be high without correlating with increased T/N ratio of the FLT $(r=0.53, P=0.014)$ because the oligodendroglioma components were included (blue circle). The T/N ratio of the MET tended to be low without correlating with increased T/N ratio of the FLT $(r=0.12, P=0.11)$ because the necrotic components were included (green circle). A significant correlation is observed between the individual T/N ratio of FDG and FLT in the grade IV gliomas $(r=0.54, P=0.05)$ (i). Between FDG and FLT, T/N ratio of the FDG tended to be high with correlating with low T/N ratio of the FLT showing a tendency toward the focus of the epileptic changes (Figures $3(\mathrm{~g})$ and $3(\mathrm{~h})$ black circle). The T/N ratio of the FDG tended to be low without correlating with increased $\mathrm{T} / \mathrm{N}$ ratio of the FLT, because the necrotic components were included in the grade IV gliomas (Figure 3(i), green circle).

Mixed neuronal and glial tumors and gliomas which easily cause convulsions had decreased accumulation of FDG until now $[26,27]$. In our case, accumulation of FDG was high in low-grade glioma which is frequently associated with convulsions. It seems that additional attention is necessary regarding the convulsive cases and accumulation of FDG.

Recently, MET and FLT have been used as tracers with tumor specificity. MET is one of the essential amino acids and a tracer for evaluating protein synthesis. FLT is a tracer of labeled thymidine for evaluating DNA synthesis, and its accumulation is considered to reflect tumor cell proliferation activity $[9,28,29]$. In the present study, there were significant differences between the grade II gliomas and the grade IV gliomas and no significant differences between the grade II gliomas and grade III gliomas in the MET-PET study.
However, the presence of tumor cells was confirmed in the area with increased accumulation of MET but poorly depicted on MRI scan and no increased accumulation of tracers in the FDG-PET and FLT-PET studies, indicating its usefulness as a testing modality to determine the presence of tumor. On the other hand, the correlation of MET uptake in the microvessel density and blood volume in tumor or with expression of vascular endothelial cells, which are amino acid transporters (LAT1) in tumor cells is suggested, and the use of MET by itself seems difficult for making diagnosis since additional factors other than proliferation activity are involved $[8,30]$. Also, it has been reported that the accumulation of MET is high in oligodendroglioma due to the high cell density, and special attention is required for its differentiation [22]. 


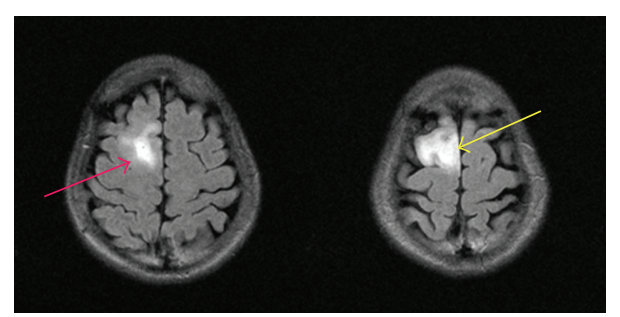

(a)

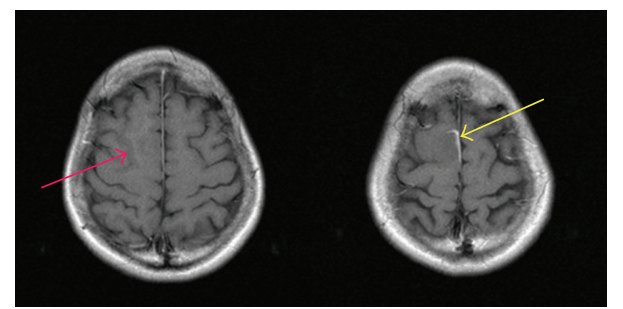

(b)

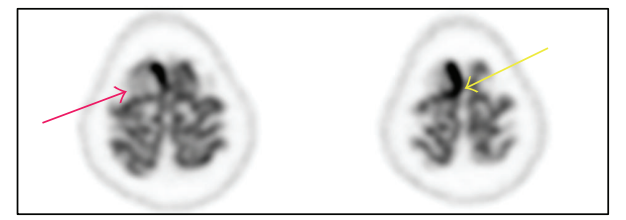

(c)

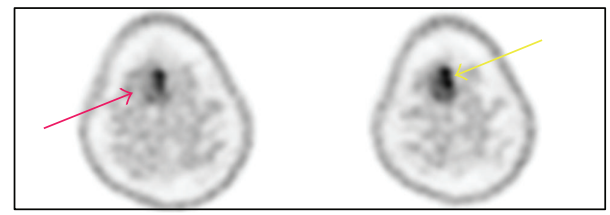

(d)

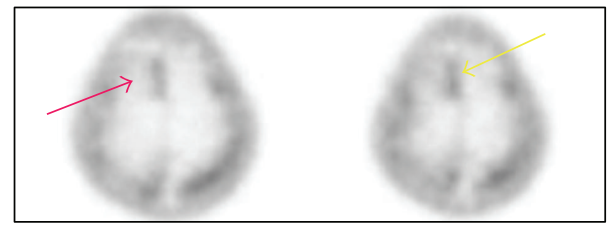

(e)

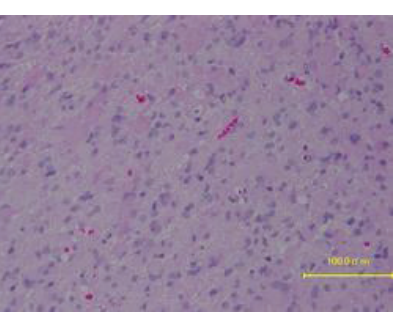

(f)

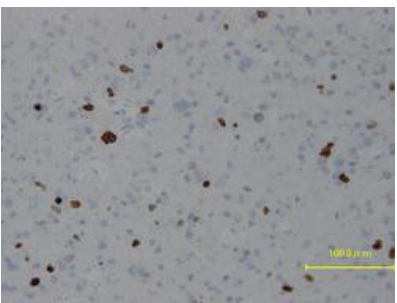

(g)

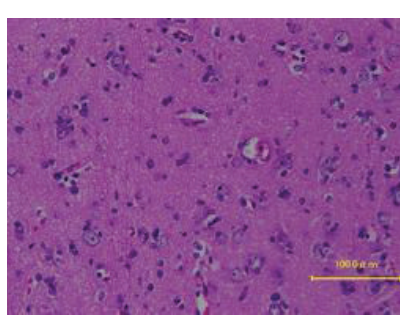

(h)

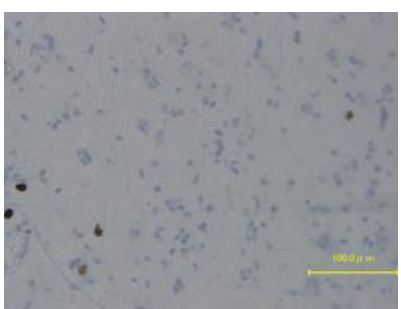

(i)

FIGURE 4: A 31-year-old woman with high intensity in the right frontal lobe in FLAIR MRI (a) and slightly enhanced lesion in Gd-enhanced MRI (b). FDG-PET demonstrated increased FDG uptake within the tumor (c). MET-PET demonstrated increased MET uptake within the tumor (d). FLT-PET demonstrated increased FLT uptake within the tumor (e). Areas of different enhancement and uptake between FDG, MET, and FLT are projected on the tumor, in order to perform histological sampling for further correlation during the resection (yellow arrow, red arrow). Yellow arrow demonstrates anaplastic astrocytoma (f) within T/N ratio of FDG-PET (2.85), MET-PET (4.57), and FLTPET (9.65). Ki-67 index from the specimen indicated by yellow arrow was $20 \%$ (g). Red arrow demonstrates diffuse astrocytoma area (h) within T/N ratio of FDG-PET (0.76), MET-PET (2.08), and FLT-PET (4.65). Ki-67 index from the specimen indicated by red arrow was $8 \%$ (i).

In the FLT-PET study, there were significant differences between the grade II gliomas and the grade IV gliomas and between the grade III gliomas and grade IV gliomas. In addition, there was a significant correlation between the Ki67 index and the FLT accumulation. There is a correlation between the Ki-67 index and the MET accumulation, but the correlation with FLT is more significant. Hence, we considered that FLT-PET was a more ideal and attractive PET tracer for glioma imaging than MET-PET. Furthermore, the uptake of FLT in normal brain tissue was lower than that of MET (SUV max, $0.20 \pm 0.05$ versus $1.52 \pm 0.36$ ). It means that FLT-PET provides a low-background cerebral image. One of the reasons for low uptake of FLT in normal brain tissue is that FLT allows the direct measurement of cellular TK1 activity, which has been proportional to the proliferation activity. Since, MET accumulation provides an indirect measure of LAT1 expression in the vascular endothelial cells and proliferation status as amino acid uptake, MET-PET exhibits higher background than FLT-PET in normal brain tissue. However, FLT-PET had some weak points. In our series of WHO grade II glioma, uptake of FLT was lower than that of MET (SUV max, $0.36 \pm 0.23$ versus $3.04 \pm 1.56$ ) and the uptake ratios for tumor to normal brain were same $(\mathrm{T} / \mathrm{N}$ ratio, $1.99 \pm 1.37$ versus $2.03 \pm 1.02)$. WHO grade II 


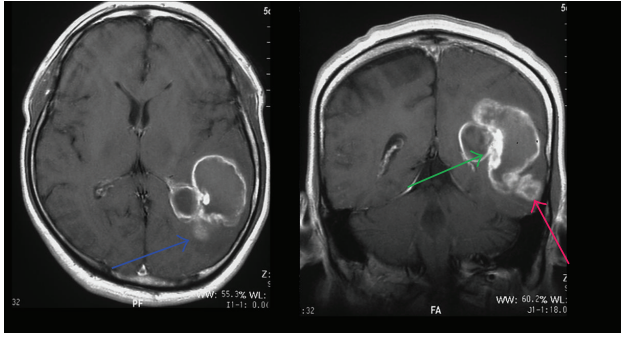

(a)

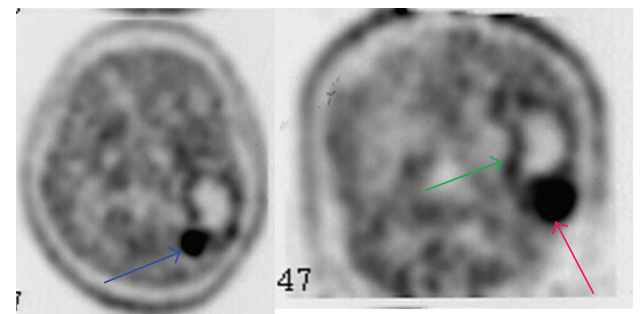

(b)

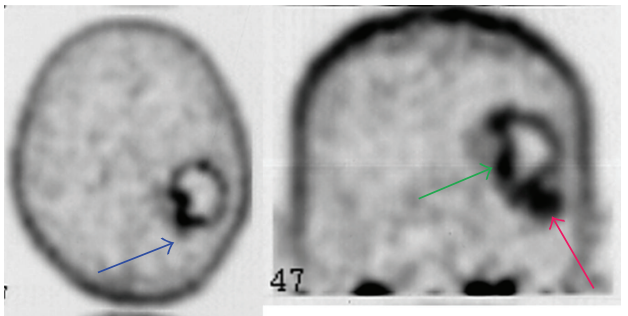

(c)

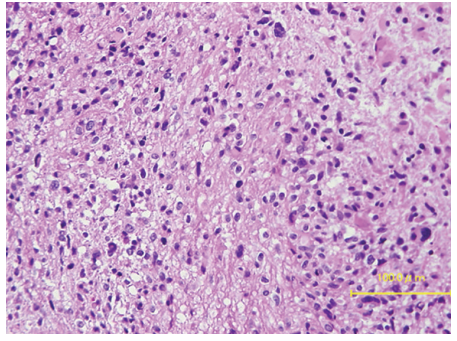

(d)

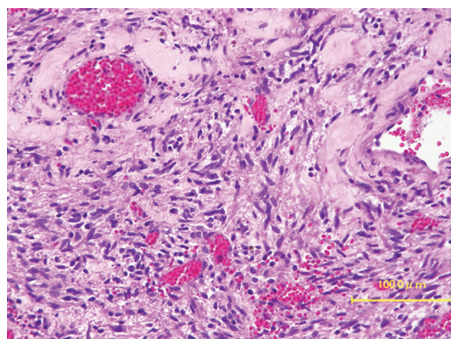

(f)

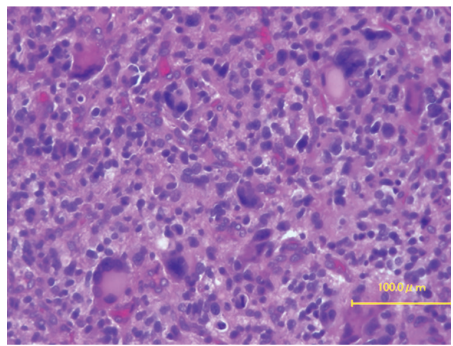

(h)

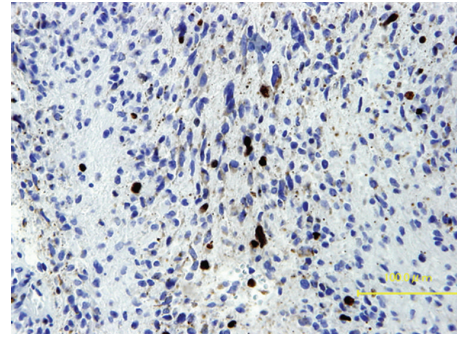

(e)

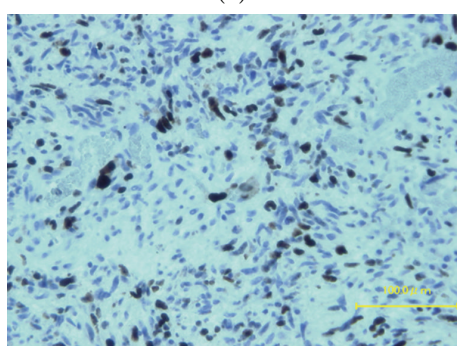

(g)

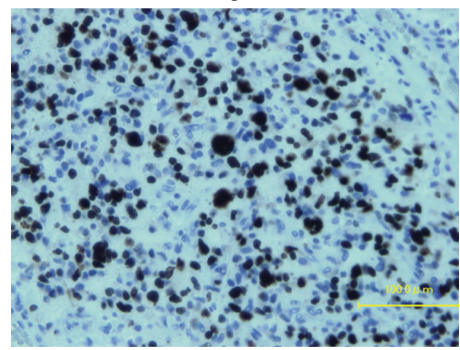

(i)

FIGURE 5: A 51-year-old man with glioblastoma. MRI clearly showed a ringed enhancement in the left temporo-occipital lobe (a). Areas of different enhancement and uptake between MET (b) and FLT (c) are projected on the tumor, in order to perform histological sampling for further correlation during the resection (blue arrow, green arrow, red arrow). Sample from the blue arrow demonstrates glioblastoma with oligodendroglioma (d) within high T/N ratio of MET-PET (4.31) and low T/N ratio of FLT-PET (3.24). Sample from the green arrow demonstrates necrosis area (f) within low T/N ratio of MET-PET (1.60) and high T/N ratio of FLT-PET (5.72). Sample from the red arrow demonstrates glioblastoma with multiple nuclear and large cells (h) within high T/N ratio of MET (3.27) and FLT-PET (5.28). Ki-67 index was $30 \%$ for the blue arrow (e), less than $10 \%$ for the green arrow (g), and $70 \%$ for the red arrow (i). Taken together, increased accumulation of both tracers (MET and FLT) appears to indicate high-grade malignancy.

gliomas had no contrast enhancement on Gd-enhanced MRI. FLT uptake was similar to the low levels observed in no enhanced lesions of MRI. FLT may be less useful in assessing proliferation in noncontrast-enhancing tumors regardless of the histopathology grading. Low-tracer access to the tumor tissue may limit the metabolic trapping of FLT after the phosphorylation even in proliferative tumors. The leakage of FLT was observed in the area with radiation necrosis and disrupted blood-brain barrier (BBB) that result in the increased accumulation $[5,24,27]$. We will continue the analysis of FLT with more patients as FLT can be quantified by kinetic analysis. Specifically, the kinetic analysis of FLTPET may clarify whether the transport effect or metabolic trapping largely contribute to the increased accumulation of FLT in the tumor. As shown in Figure 5, there were differences in the accumulation of each tracer within the same tumor due to the characteristics of each tracer, which may reflect the differences in tissues by site. In other words, we can predict that there are many oligodendroglioma components in the area with high MET accumulation and low FLT accumulation, and it is likely that the area is necrotic when MET accumulation is low, but FLT accumulation is high. When both tracer accumulations are high, the area is highly malignant as a tumor site and has high proliferative ability. However, it is difficult to make differential diagnosis, determine the recurrence of tumor, and determine the presence or absence of radiation necrosis using only one type of PET studies $[31,32]$. Our study indicated that the combinational uses of FDG, MET, and FLT-PET help us evaluate the differences in malignancy grade of the tissues and predict the evaluation of histopathological components. Furthermore, these analyses will be useful for preoperative planning of excision area.

\section{Conclusion}

PET studies using FDG, MET and FLT were conducted on 54 glioma patients. We can predict that there are many oligodendroglioma components in the area with high MET accumulation and low FLT accumulation, and it is likely 
that the area is necrotic when MET accumulation is low but FLT accumulation is high. When both MET and FLT tracer accumulations are high, the area is highly malignant as a tumor site and has high proliferative ability. PET study is a noninvasive examination method, and the use of these tracers in addition to widely used FDG-PET is highly useful for accurate preoperative diagnosis of tumors and identification of tumor area for removal. We will continue these tracer analyses to elucidate the correlation with pathological diagnosis and radiological diagnosis.

\section{Acknowledgment}

The authors appreciate the excellent technical support of the radiological technologist at their institution.

\section{References}

[1] P. J. Kelly, C. Daumas-Duport, D. B. Kispert, B. A. Kall, B. W. Scheithauer, and J. J. Illig, "Imaging-based stereotaxic serial biopsies in untreated intracranial glial neoplasms," Journal of Neurosurgery, vol. 66, no. 6, pp. 865-874, 1987.

[2] H. J. Aronen, F. S. Pardo, D. N. Kennedy et al., "High microvascular blood volume is associated with high glucose uptake and tumor angiogenesis in human gliomas," Clinical Cancer Research, vol. 6, no. 6, pp. 2189-2200, 2000.

[3] W. Chen, T. Cloughesy, N. Kamdar et al., "Imaging proliferation in brain tumors with ${ }^{18} \mathrm{~F}$-FLT PET: comparison with ${ }^{18} \mathrm{~F}$ FDG," Journal of Nuclear Medicine, vol. 46, no. 6, pp. 945-952, 2005.

[4] S. Kim, J. K. Chung, S. H. Im et al., " ${ }^{11}$ C-methionine PET as a prognostic marker in patients with glioma: comparison with ${ }^{18}$ F-FDG PET," European Journal of Nuclear Medicine and Molecular Imaging, vol. 32, no. 1, pp. 52-59, 2005.

[5] S. J. Price, "Advances in imaging low-grade gliomas," Advances and Technical Standards in Neurosurgery, vol. 35, pp. 1-34, 2010.

[6] K. Herholz, T. Hölzer, B. Bauer et al., " ${ }^{11}$ C-methionine PET for differential diagnosis of low-grade gliomas," Neurology, vol. 50, no. 5, pp. 1316-1322, 1998.

[7] L. W. Kracht, H. Miletic, S. Busch et al., "Delineation of brain tumor extent with $\left[{ }^{11} \mathrm{C}\right] \mathrm{L}$-methionine positron emission tomography: local comparison with stereotactic histopathology," Clinical Cancer Research, vol. 10, no. 21, pp. 7163-7170, 2004.

[8] S. Okubo, H. N. Zhen, N. Kawai, Y. Nishiyama, R. Haba, and T. Tamiya, "Correlation of 1 -methyl- ${ }^{11} \mathrm{C}$-methionine (MET) uptake with l-type amino acid transporter 1 in human gliomas," Journal of Neuro-Oncology, vol. 99, no. 2, pp. 217$225,2010$.

[9] S. Ceyssens, K. van Laere, T. de Groot, J. Goffin, G. Bormans, and L. Mortelmans, " $\left[{ }^{11} \mathrm{C}\right]$ methionine PET, histopathology, and survival in primary brain tumors and recurrence," American Journal of Neuroradiology, vol. 27, no. 7, pp. 14321437, 2006.

[10] T. Hatakeyama, N. Kawai, Y. Nishiyama et al., " ${ }^{11} \mathrm{C}-$ methionine (MET) and ${ }^{18} \mathrm{~F}$-fluorothymidine (FLT) PET in patients with newly diagnosed glioma," European Journal of Nuclear Medicine and Molecular Imaging, vol. 35, no. 11, pp. 2009-2017, 2008.

[11] T. Nariai, Y. Tanaka, H. Wakimoto et al., "Usefulness of L[methyl- ${ }^{11} \mathrm{C}$ ] methionine-positron emission tomography as a biological monitoring tool in the treatment of glioma," Journal of Neurosurgery, vol. 103, no. 3, pp. 498-507, 2005.

[12] K. Ishii, T. Ogawa, J. Hatazawa et al., "High L-methyl$\left[{ }^{11} \mathrm{C}\right]$ methionine uptake in brain abscess: a PET study," Journal of Computer Assisted Tomography, vol. 17, no. 4, pp. 660-661, 1993.

[13] M. Nakagawa, Y. Kuwabara, M. Sasaki et al., " ${ }^{11}$ C-methionine uptake in cerebrovascular disease: a comparison with ${ }^{18} \mathrm{~F}-$ FDG PET and 99mTc-HMPAO SPECT," Annals of Nuclear Medicine, vol. 16, no. 3, pp. 207-211, 2002.

[14] S. J. Choi, J. S. Kim, J. H. Kim et al., “[ $\left.{ }^{18} \mathrm{~F}\right] 3^{\prime}$-deoxy-3'fluorothymidine PET for the diagnosis and grading of brain tumors," European Journal of Nuclear Medicine and Molecular Imaging, vol. 32, no. 6, pp. 653-659, 2005.

[15] T. Saga, H. Kawashima, N. Araki et al., "Evaluation of primary brain tumors with FLT-PET: usefulness and limitations," Clinical Nuclear Medicine, vol. 31, no. 12, pp. 774-780, 2006.

[16] A. F. Shields, J. R. Grierson, B. M. Dohmen et al., "Imaging proliferation in vivo with [F-18]FLT and positron emission tomography," Nature Medicine, vol. 4, no. 11, pp. 1334-1336, 1998.

[17] L. B. Been, A. J. H. Suurmeijer, D. C. P. Cobben, P. L. Jager, H. J. Hoekstra, and P. H. Elsinga, "[ ${ }^{18}$ F]FLT-PET in oncology: current status and opportunities," European Journal of Nuclear Medicine and Molecular Imaging, vol. 31, no. 12, pp. 16591672, 2004.

[18] A. H. Jacobs, A. Thomas, L. W. Kracht et al., "18 F-fluoro-Lthymidine and ${ }^{11} \mathrm{C}$-methylmethionine as markers of increased transport and proliferation in brain tumors," Journal of Nuclear Medicine, vol. 46, no. 12, pp. 1948-1958, 2005.

[19] W. Chen, S. Delaloye, D. H. S. Silverman et al., "Predicting treatment response of malignant gliomas to bevacizumab and irinotecan by imaging proliferation with $\left[{ }^{18} \mathrm{~F}\right]$ fluorothymidine positron emission tomography: a pilot study," Journal of Clinical Oncology, vol. 25, no. 30, pp. 4714-4721, 2007.

[20] K. Ishiwata, C. Kasahara, K. Hatano, S. I. Ishii, and M. Senda, "Carbon-11 labeled ethionine and propionine as tumor detecting agents," Annals of Nuclear Medicine, vol. 11, no. 2, pp. 115-122, 1997.

[21] K. Roessler, B. Gatterbauer, A. Becherer et al., "Surgical target selection in cerebral glioma surgery: linking methionine (MET) PET image fusion and neuronavigation," Minimally Invasive Neurosurgery, vol. 50, no. 5, pp. 273-280, 2007.

[22] K. Torii, N. Tsuyuguchi, J. Kawabe, I. Sunada, M. Hara, and S. Shiomi, "Correlation of amino-acid uptake using methionine PET and histological classifications in various gliomas," Annals of Nuclear Medicine, vol. 19, no. 8, pp. 677-683, 2005.

[23] C. Colavolpe, E. Guedj, P. Metellus et al., "FDG-PET to predict different patterns of progression in multicentric glioblastoma: a case report," Journal of Neuro-Oncology, vol. 90, no. 1, pp. 47-51, 2008.

[24] K. Herholz, D. Coope, and A. Jackson, "Metabolic and molecular imaging in neuro-oncology," Lancet Neurology, vol. 6, no. 8, pp. 711-724, 2007.

[25] C. La Fougère, B. Suchorska, P. Bartenstein, F. W. Kreth, and J. C. Tonn, "Molecular imaging of gliomas with PET: opportunities and limitations," Neuro-Oncology, vol. 13, no. 8, pp. 806-819, 2011.

[26] R. Talanow, P. Ruggieri, A. Alexopoulos, D. Lachhwani, and $\mathrm{G}$. Wu, "PET manifestation in different types of pathology in epilepsy," Clinical Nuclear Medicine, vol. 34, no. 10, pp. 670674, 2009.

[27] J. H. Phi, J. C. Paeng, H. S. Lee et al., "Evaluation of focal cortical dysplasia and mixed neuronal and glial tumors in 
pediatric epilepsy patients using ${ }^{18} \mathrm{~F}-\mathrm{FDG}$ and ${ }^{11} \mathrm{C}$-methionine pet," Journal of Nuclear Medicine, vol. 51, no. 5, pp. 728-734, 2010.

[28] N. Kawai, T. Hatakeyama, T. Tamiya et al., "Is it possible to differentiate between radiation necrosis and recurrence of brain tumors using positron emission tomography?" Progress in Computed Imaging, vol. 30, no. 1, pp. 1-11, 2008.

[29] N. Kawai, M. Kagawa, T. Hatakeyama et al., "11 C-methionine positron emission tomography in brain tumor," Neurological Surgery, vol. 36, no. 10, pp. 847-859, 2008.

[30] Y. Okita, M. Kinoshita, T. Goto et al., " ${ }^{11} \mathrm{C}$-methionine uptake correlates with tumor cell density rather than with microvessel density in glioma: a stereotactic image-histology comparison," NeuroImage, vol. 49, no. 4, pp. 2977-2982, 2010.

[31] Y. Terakawa, N. Tsuyuguchi, Y. Iwai et al., "Diagnostic accuracy of ${ }^{11} \mathrm{C}$-methionine PET for differentiation of recurrent brain tumors from radiation necrosis after radiotherapy," Journal of Nuclear Medicine, vol. 49, no. 5, pp. 694-699, 2008.

[32] M. Kinoshita, T. Goto, H. Arita et al., "Imaging ${ }^{18} \mathrm{~F}$ fluorodeoxy glucose $/{ }^{18} \mathrm{C}$-methionine uptake decoupling for identification of tumor cell infiltration in peritumoral brain edema," Journal of Neuro-Oncology, vol. 106, no. 2, pp. 417425, 2012. 


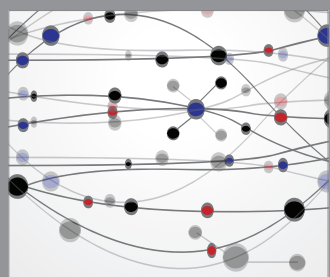

The Scientific World Journal
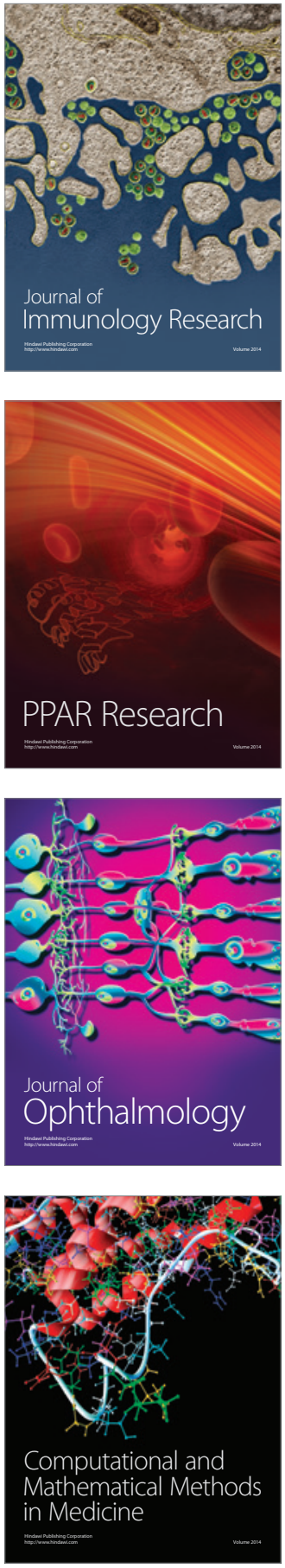

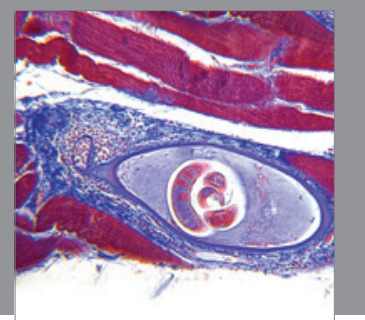

Gastroenterology

Research and Practice
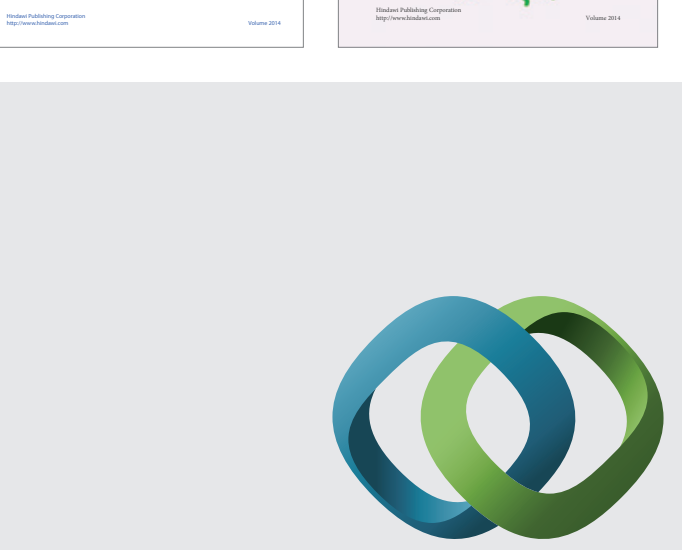

\section{Hindawi}

Submit your manuscripts at

http://www.hindawi.com
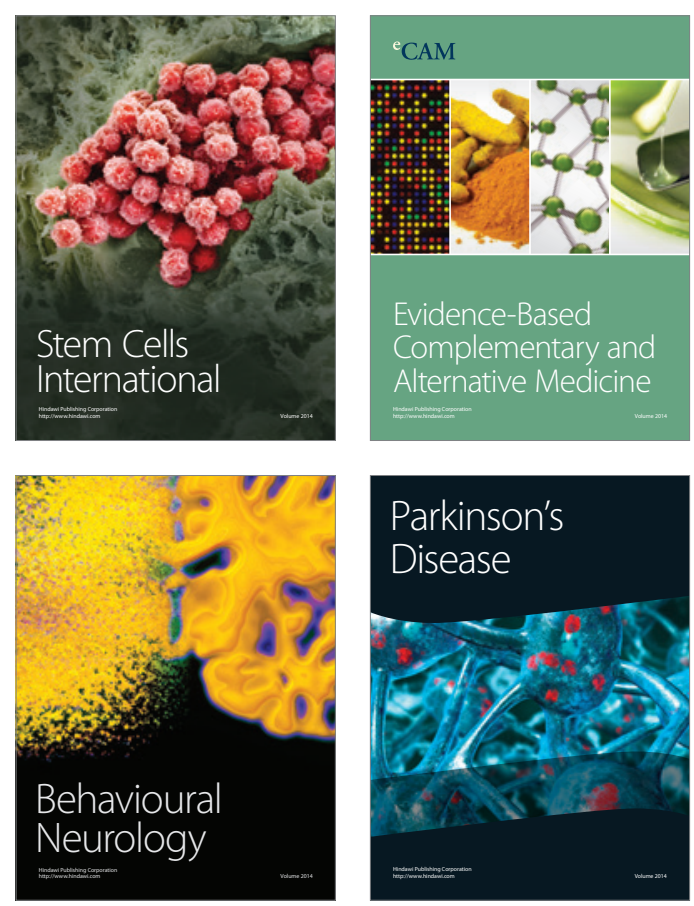

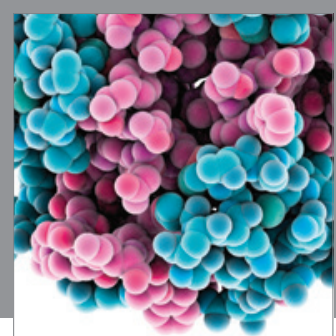

Journal of
Diabetes Research

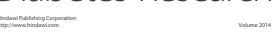

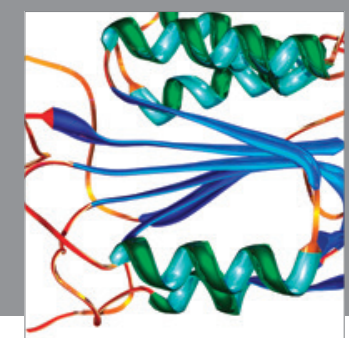

Disease Markers
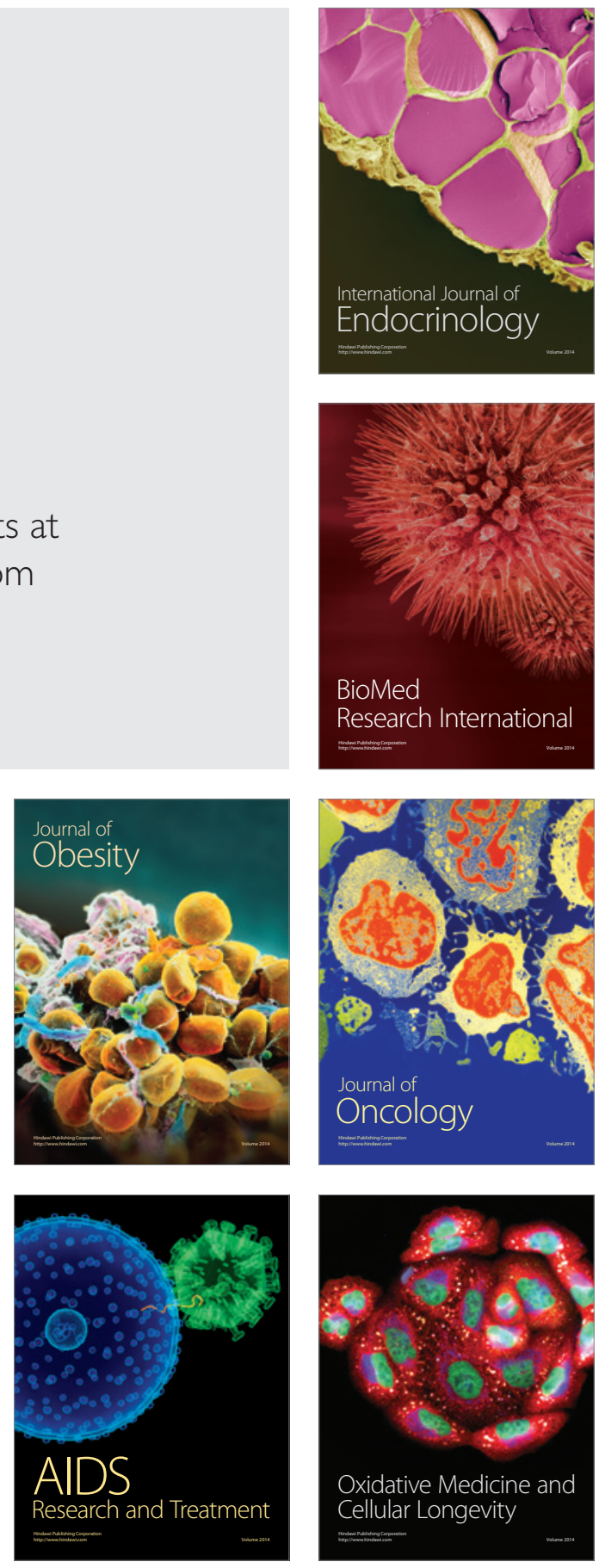